가돌리늄 조영증강 자기공명영상과 확산강조영상의 간신경내분비종양 진단능력에 대한 비교연구

장석기 $\left.\right|^{1} \cdot$ 김정훈 $^{2,3} \cdot$ 유미혜 ${ }^{4} \cdot$ 한준구 $^{2,3}$

${ }^{1}$ 분당제생병원 영상의학과, 서울대학교 의과대학 ${ }^{2}$ 영상의학교실, ${ }^{3}$ 방사선의학연구소, ${ }^{4}$ 건국대학교 의학전문대학원 영상의학교실

\title{
Diagnostic Performance of Diffusion-weighted Imaging for Hepatic Neuroendocrine Tumor: Comparison with Combined Diffusion-weighted Imaging and Contrast-enhanced Magnetic Resonance Imaging
}

Suk Ki Jang ${ }^{1}$, Jung Hoon $\mathrm{Kim}^{2,3}$, Mi Hye Yư ${ }^{4}$, Joon Koo Han ${ }^{2,3}$

'Department of Radiology, Bundang Jesaeng Hospital, Seongnam; Departments of ${ }^{2}$ Radiology, ${ }^{3}$ Institute of Radiation Medicine, Seoul National University College of Medicine; ${ }^{4}$ Department of Radiology, Konkuk University School of Medicine, Seoul, Korea

Received May. 18, 2016

Revised Jun. 28, 2016

Accepted Jun. 28, 2016
Background/Aims: To investigate the diagnostic performance of diffusion-weighted imaging (DWI) for hepatic neuroendocrine tumors (NET) compared with combined DWI and contrastenhanced magnetic resonance imaging (MRI) .

Methods: Fifteen patients with hepatic NET ( $n=128)$ underwent enhanced MRI and DWI with multiple-b values. We analyzed three different sets: Precontrast set; DWI set (added DWI); combined set (added enhanced image). Two reviewers rated possibility of NET using a 5-point scale for each image set. Their diagnostic performance was compared using Jackknife alternative free-response ROC (JAFROC).

Results: Diagnostic performance was better on the combined set (figure of merit $[\mathrm{FOM}]=0.852$, $0.761)$ than the precontrast set $(\mathrm{FOM}=0.427,0.572, P<0.05)$ and the $\mathrm{DWI}$ set $(\mathrm{FOM}=0.682$, $0.620, P<0.05)$. However, DWI improved performance compared with precontrast set without statistical difference. In small NETs $(<1 \mathrm{~cm})$, all sets showed low sensitivity (10.7-65.9\%) with high specificity (95.4-100\%). Interobserver agreement was moderate in all image sets $(k=0.521$ to 0.589).

Conclusions: Combined DWI and enhanced MRI were more useful for detecting NET. Although statistically insignficant, there was a trend in improved diagnostic performance with DWI. (J Liver Cancer 2016;16:92-100)

Keywords: Diffusion-weighted imaging; Hepatic neuroendocrine tumor; Contrast-enhanced MRI; Comparison study 


\section{INTRODUCTION}

The liver is the most common site of metastatic disease in neuroendocrine tumors (NET). ${ }^{1}$ The presence of liver metastases has been shown to be a major factor altering both the patient's quality of life and prognosis, regardless of the primary site. ${ }^{2}$ Therefore, early tumor detection is very important.

Magnetic resonance imaging (MRI) is considered to be the most successful method for detecting liver metastases. ${ }^{3} \mathrm{Gad}-$ olinium-enhanced MRI is essential for the diagnosis of hepatic metastasis. However, gadolinium-based contrast agents seem to be an important trigger for the development of nephrogenic systemic fibrosis (NSF) in patients with severe renal impairment, and there is currently no uniformly effective treatment for NSF. ${ }^{4}$ Therefore, for patients with renal impairment, physicians should carefully consider the use of gadolinium-based contrast agents.

Diffusion-weighted imaging (DWI) has a potential role in the detection and characterization of liver tumors based on the high contrast between a lesion and normal tissue. ${ }^{5,6}$ Recent advances in MR technology, such as high magnetic fields of 3-Tesla, high-performance gradient coils, and parallel imaging techniques have allowed further improvement in the abdominal DWI quality by increasing the signal-to-noise ratio, reducing blurring, and minimizing susceptibility-induced artifacts. ${ }^{5,7,8}$ DWI can be performed quickly and can be easily incorporated into a standard clinical protocol. Nasu et al. ${ }^{9}$ reported that the combined reading of DWI and unenhanced MRI results in a higher accuracy for the detection of hepatic metastases. According to previous reports, low b-value DWI appears to be a better method for detecting liver lesions and a high b-value may help in lesion characterization. ${ }^{10}$ Moreover, recently developed magnetic resonance (MR) techniques have made multi-b value DWI possible. ${ }^{11}$

To our knowledge, there has been no previous study focusing on the diagnostic performance of DWI for hepatic NET compared to combined DWI and contrast-enhanced MRI. Therefore, the purpose of our study is to compare the diagnostic performance of DWI for hepatic NET lesions using combined DWI and contrast-enhanced MRI.

\section{METHODS}

\section{Patient population}

Our institutional review board approved this retrospective study, and the requirement for informed consent was waived. Between October 2008 and March 2012, 43 patients with confirmed neuroendocrine tumor underwent contrast-enhanced MR imaging and DWI for suspected hepatic neuroendocrine tumor metastasis. Among these patients, 15 patients were considered ineligible for study participation due to more than 15 liver metastases in a single patient $(n=8)$ or no significant hepatic focal lesion seen on MRI $(n=7)$. Of the 28 patients included in our study, 15 had 128 hepatic NETs, average 9 lesions per patient, range from 2 to 15 lesions per patient, and 13 patients had 29 benign hepatic lesions including hepatic cyst $(n=21)$ and hemangioma $(n=8)$. Of the 128 hepatic NETs, 93 were $10 \mathrm{~mm}$ or less in diameter, and the remaining 35 ranged from 11 to $91 \mathrm{~mm}$ in diameter. The mean hepatic NET size was $9.10 \pm 11.92 \mathrm{~mm}$ (range, 2-98 mm). Our study population consisted of 18 males and 10 females (M: $\mathrm{F}=1.8: 1)$ with an age range of 25-75 years (mean age and SD, $56.8 \pm 11.7$ years). The primary tumor sites were the pancreas $(n=22)$, liver $(n=3)$, stomach $(n=2)$, and the Ampulla of Vater $(n=1)$.

The reference standard for confirming the neuroendocrine tumor was based on a histopathological examination of the surgical or biopsy specimens. Twenty-three patients underwent surgery, i.e. pylorus-preserving pancreaticoduodenectomy $(n=11)$, Whipple surgery $(n=1)$, partial pancreatectomy $(n=8)$, subtotal gastrectomy $(n=1)$, and partial hepatectomy $(n=2)$. Five patients underwent liver biopsy. The diagnosis of hepatic NET depended on a combination of the typical image findings of hepatic NET, i.e. hypointensity on T1-weighted images, hyperintensity on T2-weighted images, marked arterial enhancement on the early dynamic phase, typical wash-out on the portal-venous phase, and interval growth with the same enhancement pattern seen on follow-up dynamic liver computed tomography (CT) or MRI with a mean follow-up period of 20.5 months (range 6-57 months). There was no histopathological confirmation 
for the benign hepatic lesions. We considered lesions as benign when they showed typical findings and stability on follow-up imaging examinations during a mean follow-up period of 22 months (range 6-55 months). Hemangiomas ( $\mathrm{n}=8$ ) and cysts $(n=21)$ were diagnosed based on typical imaging findings, such as peripheral nodular enhancement and progressive fill-in seen on dynamic MRI and bright hyperintensity similar to that of cysts seen on T2-weighted images for hemangiomas and homogeneous signal intensities similar to that of water and no enhancement for cysts.

\section{MRI protocol}

MRI was performed with one 1.5-T system (Signa Excite HDX, GE Medical Systems, Milwaukee, WI, USA, n=6) or one of two 3-T superconducting systems (Verio, Siemens Medical Solutions, Erlangen, Germany, $n=20$; Intera Achieva, Philips Healthcare, Best, Netherlands, $\mathrm{n}=2$ ) using an 8-channel (HDX), a 32-channel (Verio) or a 6-channel (Achieva) phased-array torso coil. The MR parameters of each sequence are summarized in Appendix 1. Our MR protocol for the pancreas included transverse T1-weighted imaging (T1WI) including in-phase and opposed-phase spoiled gradient echo (GRE) techniques, fat-saturated T1-weighted GRE images, and transverse T2-weighted imaging (T2WI) including fat-saturated $\mathrm{T} 2$-weighted fast spin-echo images or ultrafast T2-weighted fast spin-echo (HASTE) images. Diffusion-weighted imaging (DWI) was obtained using multisection, single-shot, spin-echo, echo-planar imaging with a spectral presaturation attenuated inversion-recovery (SPAIR), fat-suppressed pulse sequence during free-breathing scanning with a body coil and using the following imaging parameters: TR/TE, 2,300-5,000/40-70; ETL, 1; flip angle, 90 ; FOV, 380-380 mm; section thickness, $7 \mathrm{~mm}$; matrix, 160 $\times 160$; and with b values of $0,50,400$, and $800 \mathrm{~s} / \mathrm{mm}^{2}$. All separate image series were acquired with diffusion weighting in the axial direction using three-directional diffusion gradients.

Dynamic images were obtained using a fat-suppressed, 3D GRE sequence (liver acquisition with volume acceleration, LAVA, GE Medical Systems; volume interpolation with breath-hold examination, VIBE, Siemens Medical Solutions; T1-weighted, high-resolution, isotropic-volume examination, THRIVE, Philips Healthcare) before and after administration of gadopentetate dimeglumine (Magnevist, BayerSchering Pharma, Berlin, Germany or Multi-Hance, Bracco, Milan, Italy; $\mathrm{n}=18$ ) at a dose of $0.1 \mathrm{mmol}$ per kilogram of body weight and with an injection rate of $2 \mathrm{~mL} / \mathrm{sec}$ or of gadoxetic acid (Primovist, Bayer-Schering Pharma, Berlin, Germany; $\mathrm{n}=10)$ as a bolus of $0.1 \mathrm{~mL} / \mathrm{kg}(0.025 \mathrm{mmol}$ per kilogram) at a rate of $1.5 \mathrm{~mL} / \mathrm{sec}$ using a power injector (Spectris Solaris EP; Medrad, Warrendale, PA, USA), followed by a $25-\mathrm{mL}$ saline flush. Acquisition of 3D-LAVA, VIBE or THRIVE data for each phase was completed during a single breath-hold at the end of expiration (time range 1822 seconds, mean time 21 seconds). Arterial, portal-venous, and equilibrium-phase images were obtained serially at 2040 seconds, 45-70 seconds, and three minutes, respectively, after contrast injection. All sequences were obtained in the axial plane.

\section{Image interpretation and analysis}

Two radiologists (JHK, MHY) specializing in abdominal imaging and with 16 and six years of clinical experience, respectively, and who were blinded to the laboratory and histological data, reviewed all of the MR images on a picture archiving and communication system (PACS) workstation monitor. MR images were interpreted independently and in random order by the two radiologists for the presence or probability of hepatic NET. In the MR interpretation session, three sets of MR images were provided sequentially for each patient, i.e. the precontrast set (precontrast T1- and T2weighted images); DWI set (precontrast image set with added DWI); and the combined image set (DWI set with the added, contrast-enhanced image). DWI was interpreted using all those b-value images. In order to minimize any learning bias, the readers evaluated the three image sets with an interval of at least one week. Each reader documented the presence and location of the hepatic lesions and assigned each lesion a fivepoint confidence scale: 1 , definitely benign; 2 , probably benign; 3 , indeterminate; 4 , probable metastasis; 5 , definite me- 
tastasis. The sensitivity calculations were based only on those lesions assigned a confidence rating of 4 or 5 for metastasis. When a lesion was detected, its location, size, and confidence score were reported on the review sheet so that the study coordinators could identify the lesions demonstrated by the reader (lesion-by-lesion analysis). If any false-positive or false-negative results were observed in any reader's interpretation, the study coordinators also assessed the reason for the misinterpretation by reviewing the CT and MR images. One radiologist (SKJ) measured the largest tumor diameter of the liver metastasis three times on contrast enhanced MRI.

\section{Statistical Analysis}

All lesions, i.e. small lesions equal to less than $1 \mathrm{~cm}$ in diameter and large lesions over $1 \mathrm{~cm}$ in diameter, were analyzed separately. Jackknife alternative free-response ROC (JAFROC), especially developed to analyze observer free-response tasks, was used to compare the diagnostic performance of MR imaging for depicting hepatic NET. JAFROC analysis has been proposed for estimating statistically significant differences between modalities when location issues are relevant. JAFROC analysis is based on a free-response receiver operating characteristic (FROC) paradigm and accounts for reader variation. Conventional receiver operating characteristic (ROC) analysis is of limited value for this type of application because only one signal can be used per case, and the location of the signal cannot be taken into account in the evaluation. In contrast, FROC analysis allows evaluation of the radiologists' performance in diagnosing medical images using multiple responses, each with information regarding the confidence level and location. Jackknife FROC software (JAFROC, version 4.1; http://www.devchakraborty.com) $)^{12,13}$ was used to compute the figure of merit (FOM) values (the analogue of the area under the ROC curve) for each reader. The FOM is defined as the probability that a true-positive lesion is rated higher than the highest rated false-positive lesion on normal images. Normal images with no marks and unmarked lesions are assigned a zero rating. To calculate the sensitivity, specificity, positive predictive value (PPV), and negative predictive value (NPV) for each imaging modality, lesions assigned to grades 4 or 5 were regarded as positive for the presence of hepatic NET. The kappa values were calculated to assess the inter-reader variability for detecting pancreatic carcinoma and liver metastasis. A kappa value of 0.81 1.00 was interpreted as excellent; 0.61-0.80, substantial agreement; 0.41-0.60, moderate agreement; 0.21-0.40, fair agreement; and 0.00-0.20, poor agreement. All statistical analysis was performed using MedCalc (v.8.0.0.1 for Windows; MedCalc Software, Mariakerke, Belgium). A $P$-value of 0.05 or less was considered to indicate a statistically significant difference.

\section{RESULTS}

The diagnostic performance for detecting hepatic NET was

Table 1. Diagnostic performance for detection of hepatic NET: lesion-by-lesion analysis

\begin{tabular}{|c|c|c|c|c|c|c|}
\hline & \multicolumn{3}{|c|}{ Lesion-by-Lesion Analysis: all lesions } & \multicolumn{3}{|c|}{$P$-value } \\
\hline & A & B & C & A vs. $B$ & B vs. C & A vs. C \\
\hline \multicolumn{7}{|l|}{ All $(n=128)$} \\
\hline Reviewer 1 & 0.427 & 0.682 & 0.852 & $<0.001$ & 0.003 & $<0.001$ \\
\hline Reviewer 2 & 0.572 & 0.620 & 0.761 & 0.433 & 0.028 & 0.004 \\
\hline \multicolumn{7}{|l|}{$>1 \mathrm{~cm}(\mathrm{n}=35)$} \\
\hline Reviewer 1 & 0.662 & 0.845 & 0.931 & 0.012 & 0.214 & 0.0007 \\
\hline Reviewer 2 & 0.628 & 0.631 & 0.839 & 0.974 & 0.026 & 0.025 \\
\hline \multicolumn{7}{|l|}{$\leq 1 \mathrm{~cm}(\mathrm{n}=93)$} \\
\hline Reviewer 1 & 0.353 & 0.632 & 0.815 & 0.0003 & 0.011 & $<0.001$ \\
\hline Reviewer 2 & 0.531 & 0.617 & 0.755 & 0.243 & 0.067 & 0.004 \\
\hline
\end{tabular}

'A' means 'Precontrast MR set', 'B' means 'DWI set', and 'C' means 'Combined image set'.

NET, neuroendocrine tumors; DWI, diffusion-weighted imaging. 
better on the combined image set $(\mathrm{FOM}=0.852$ and 0.761$)$ than on the precontrast set $(\mathrm{FOM}=0.427$ and $0.572, P<0.05)$ and the DWI set $(\mathrm{FOM}=0.682$ and $0.620, P=0.169 P<0.05)$. The diagnostic performance for the detection of hepatic NET is summarized in Table 1. DWI improved the diagnostic performance for detecting hepatic NET without statistical significance compared to precontrast set (Fig. 1). Interobserver agreement was moderate in all image sets (precontrast set, $\kappa=0.521$; DWI set, $\kappa=0.589$; and combined image set, $\kappa=0.540$ )

All image sets showed higher specificity (range, 86.21$96.55 \%$ ) with lower sensitivity (range, 21.88-71.88\%). The sensitivity and specificity for detecting hepatic NET are summarized in Table 2. The combined image set revealed higher sensitivities than the DWI set for both readers. For the combined image set, 16 hepatic NETs $(12.5 \%)$ by Reader 1 and 23 hepatic NETs $(17.18 \%)$ by Reader 2 were additionally seen when compared to the DWI set (Fig. 2). In contrast, there were three hepatic NETs that were better visualized on DWI than on combined DWI and contrast-enhanced MRI (Fig. 3).

In hepatic NETs larger than $1 \mathrm{~cm}$, the overall sensitivity for the detection of hepatic NET was increased. The sensitivity and specificity for the detection of hepatic NETs larger than $1 \mathrm{~cm}$ are summarized in Table 3 . In our study, of the 128 hepatic NETs, 93 were $10 \mathrm{~mm}$ or less in diameter, and the remaining 35 ranged from 11 to $91 \mathrm{~mm}$ in diameter. In hepatic NETs less than or equal to $1 \mathrm{~cm}$, the sensitivity for detecting them was better on the combined image set $(65.59 \%$ and $61.29 \%)$ than on the precontrast image set $(10.75 \%$ and
$15.05 \%)$ and the DWI set (50.54\% and 39.78\%) and with a higher specificity (range, 90.91-100\%). The sensitivity and specificity for the detection of hepatic NETs less than or equal to $1 \mathrm{~cm}$ are summarized in Table 4 . Most of the hepatic NETs not detected by DWI and contrast-enhanced imaging were less than $1 \mathrm{~cm}$ (mean size and SD, $0.579 \pm 0.28 \mathrm{~cm}$ ). Of the hepatic NETs missed by DWI and detected on combined DWI and contrast-enhanced imaging, nine were located directly below the diaphragm or in the left liver lobe.

\section{DISCUSSION}

According to our study results, the diagnostic performance for detecting hepatic NET was better on the combined image set $(\mathrm{FOM}=0.852$ and 0.761$)$ than on the precontrast set $(\mathrm{FOM}=0.427$ and $0.572, P<0.05)$ and the DWI set $(\mathrm{FOM}=0.682$ and 0.620, $P=0.169 P<0.05)$. Hepatic metastasis of NETs is fairly common and is encountered in $25-90 \%$ of patients with NETs. ${ }^{14}$ On contrast-enhanced MRI, hepatic NET showed an intense enhancement in the hepatic arterial-dominant phase with washout in the portal-venous and equilibrium-phase, thus reflecting the hypervascularity of these lesions. ${ }^{15,16}$ Bader et al. reported that $94 \%$ of hepatic NETs in their patients were hypervascular and that $15 \%$ were seen only on immediate, gadolinium-enhanced images. ${ }^{17}$ Another study showed that the combination of hepatic arterial-phase and fat-suppressed, fast spin-echo, T2-weighted images depicted 80\% of hepatic NETs. ${ }^{18}$ In a study by Soyer et al. the combination of unenhanced MR images and contrast-enhanced MR images al-
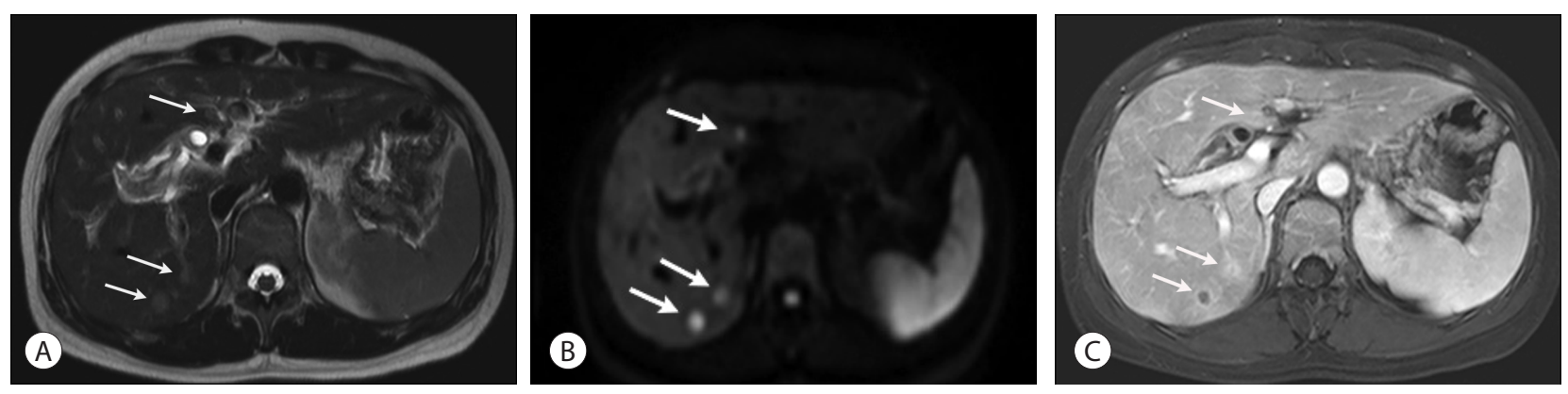

Figure 1. 33-year-old woman with biopsy confirmed hepatic NET. (A) T2-weighted image shows hyper signal intensity nodules in the right posterior segment, and a suspicious hyper signal intensity nodule in the left medial segment (arrows). (B) Diffusion weighted image using $b$ values of $800 \mathrm{~s} / \mathrm{mm}^{2}$ clearly demonstrates hyper signal intensity nodules in the right posterior segment and in the left medial segment (arrows). (C) Dynamic images using a fat-suppressed, 3D GRE sequence, on the arterial phase, clearly demonstrates peripheral rim enhancing nodules in the same location (arrows). NET, neuroendocrine tumors; GRE, gradient echo. 
lowed more accurate characterization of hepatic NETs and a clearer differentiation from cavernous hemangiomas. ${ }^{19}$ Our

Table 2. Sensitivity and specificity for detection of hepatic NET: lesion-by-lesion analysis

\begin{tabular}{lccc}
\hline & \multicolumn{2}{c}{ Lesion-by-Lesion Analysis: all lesions } \\
\cline { 2 - 4 } & $\begin{array}{c}\text { Precontrast } \\
\text { MR set }\end{array}$ & DWI set & $\begin{array}{c}\text { Combined } \\
\text { image set }\end{array}$ \\
\hline Sensitivity (\%) & & & \\
Reviewer 1 & $21.88(28 / 128)$ & $59.38(76 / 128)$ & $71.88(92 / 128)$ \\
Reviewer 2 & $27.34(35 / 128)$ & $48.44(62 / 128)$ & $66.41(85 / 128)$ \\
Specificity (\%) & & & \\
Reviewer 1 & $96.55(28 / 29)$ & $96.55(28 / 29)$ & $96.55(28 / 29)$ \\
Reviewer 2 & $93.10(27 / 29)$ & $86.21(25 / 29)$ & $89.66(26 / 29)$ \\
PPV (\%) & & & \\
Reviewer 1 & $96.55(28 / 29)$ & $98.70(76 / 77)$ & $98.92(92 / 93)$ \\
Reviewer 2 & $94.59(35 / 37)$ & $93.94(62 / 66)$ & $96.59(85 / 88)$ \\
NPV (\%) & & & \\
Reviewer 1 & $21.88(28 / 128)$ & $35.00(28 / 80)$ & $43.75(28 / 64)$ \\
Reviewer 2 & $22.50(27 / 120)$ & $27.47(25 / 91)$ & $37.68(26 / 69)$ \\
\hline
\end{tabular}

NET, neuroendocrine tumors; MR, magnetic resonance; DWI, diffusion-weighted imaging; PPV, positive predictive value; NPV, negative predictive value. study results were also similar to those noted above in which combined reading with contrast-enhanced imaging is warranted in order to ensure better detection and characterization of hepatic NETs. In particular, in liver metastases less than or equal to $1 \mathrm{~cm}(93 / 128,72.6 \%)$, the sensitivity was better on the combined image set $(65.59 \%$ and $61.29 \%)$ than on the other image sets $(10.75 \%$ and $15.05 \%$ on the precontrast image set, $50.54 \%$ and $39.78 \%$ on the DWI set). As in our study, Shimada et al. reported the higher accuracy of gadoxetic acid-enhanced MRI than that of DWI in the detection of small hepatic metastases ( $2 \mathrm{~cm}$ or less). ${ }^{5}$

However, according to our study, DWI improved the diagnostic performance for detecting hepatic NET without statistical significance compared to precontrast set. In addition, there were three hepatic NETs that were better visualized on DWI than on combined DWI and contrast-enhanced MRI. In cases of liver metastasis from other primary cancer lesions, several reports have demonstrated that DWI alone or combined reading of DWI and contrast-enhanced imaging significantly increased the diagnostic accuracy ${ }^{920-23}$. Nasu et al. reported that DWI, when evaluated in conjunction with un-
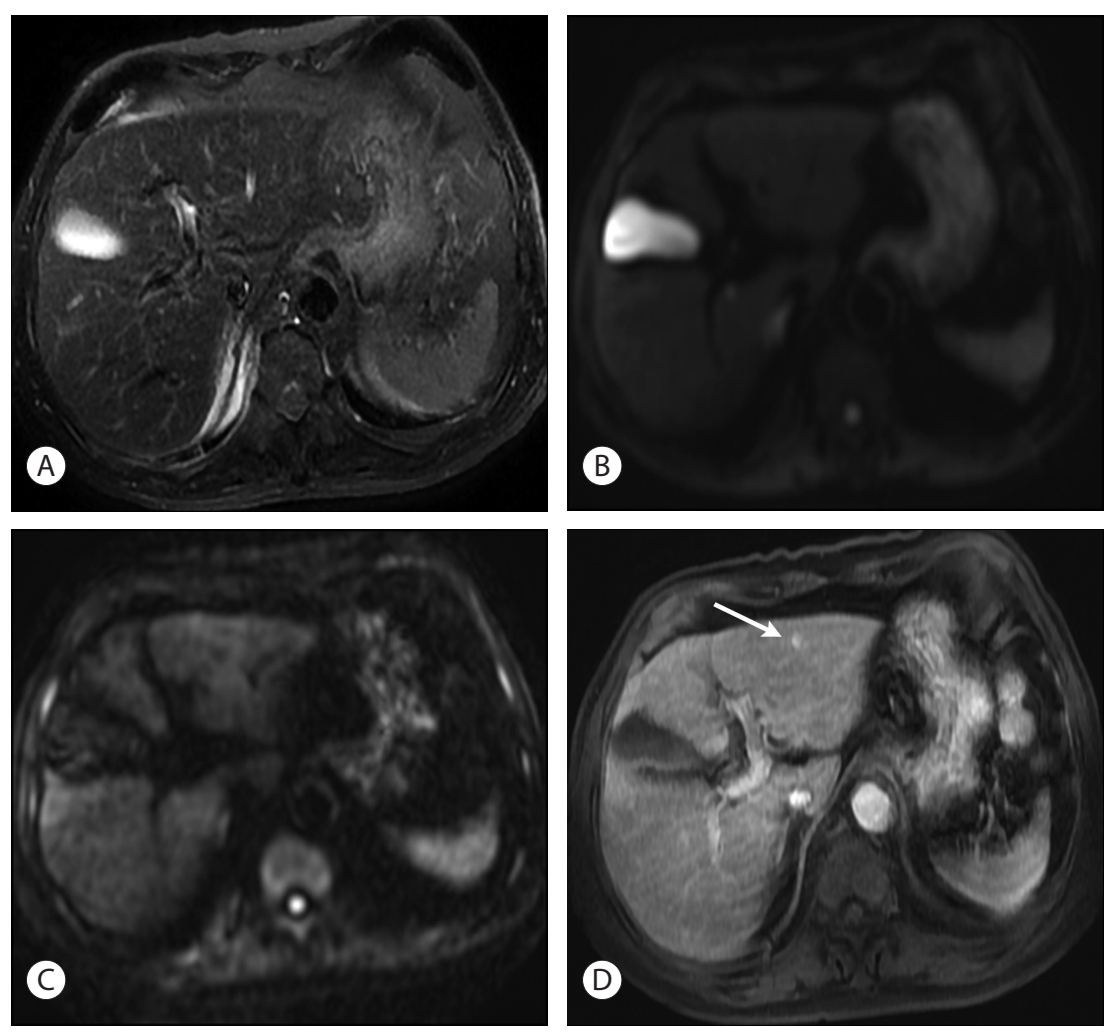

Figure 2. False negative on DWI. 78-year-old man with biopsy proven hepatic NET. (A-C) T2-weighted image and diffusion weighted image using $b$ values of $50 \mathrm{~s} / \mathrm{mm}^{2}$ and $800 \mathrm{~s} / \mathrm{mm}^{2}$ show no definite nodule in the liver. (D) Dynamic images using a fatsuppressed, 3D GRE sequence, on the arterial phase, clearly demonstrates small enhancing nodule in the lateral segment of left lobe (arrow). DWI, diffusion-weighted imaging; NET, neuroendocrine tumors; GRE, gradient echo. 
enhanced MR images, was superior to superparamagnetic iron oxide (SPIO)-enhanced MR imaging for the detection of liver metastases. ${ }^{9}$ In our study, DWI improved both the lesion detection rate and the diagnostic performance compared with the precontrast set. Compared to the background parenchyma, sustained hyperintensity was subjectively regarded as a solid malignant lesion. Based on current evidence, gadolinium-based contrast agents seem to have a role in the development of NSF in patients with renal impairment. ${ }^{4}$ We believe that DWI should have important role for diagnosing hepatic NET without added contrast material.

Despite the increased diagnostic performance of DWI, we demonstrated that there were nine, small metastases directly below the diaphragm or in the left liver lobe that were missed by DWI and were detected on contrast-enhanced imaging. On DWI, artifacts caused by physiological movement, particularly subcardiac and subphrenic signal loss, which appeared most frequently, significantly decreased the sensitivity for the detection of small hepatic metastases in the lateral segment and the upper edge of the liver. Furthermore, magnetic susceptibility artifacts, which were seen at the boundary between the lung and the liver parenchyma owing to the heterogeneity of the magnetic field, also reduced the sensitivity in the upper edge of the liver. This issue is still considered as one of the primary limitations when performing DWI of the liver. ${ }^{5}$ However, upgraded MR systems using artifact-reducing strategies will offer better DWI image quality, and thus leading to better diagnostic performance for small lesions.

This study has limitations. First, only a small number of hepatic NETs and no benign lesions were histopathologically confirmed. Obtaining a histopathologic diagnosis in all patients would be desirable, although it is not clinically and ethically appropriate in most cases. Second, as the study design is retrospective, a selection bias was inevitable. Furthermore, contrast-enhanced MRI was performed with three, different MR scanners including 1.5 T and $3 \mathrm{~T}$ systems with two, different MR contrast materials due to the retrospective study design. However, despite this heterogeneity of MRI, we used the acceptable and adequate image quality of MRI for

Table 3. Sensitivity and specificity for detection of hepatic NET: lesion-by-lesion analysis for lesions greater than $1 \mathrm{~cm}$

\begin{tabular}{lccc}
\hline & \multicolumn{2}{c}{ Lesion-by-Lesion Analysis: > cm lesion } \\
\cline { 2 - 4 } & $\begin{array}{c}\text { Precontrast } \\
\text { MR set }\end{array}$ & DWI set & $\begin{array}{c}\text { Combined } \\
\text { image set }\end{array}$ \\
\hline Sensitivity (\%) & & & \\
Reviewer 1 & $51.43(18 / 35)$ & $82.86(29 / 35)$ & $88.57(31 / 35)$ \\
Reviewer 2 & $57.14(20 / 35)$ & $71.43(25 / 35)$ & $80.00(28 / 35)$ \\
Specificity (\%) & & & \\
Reviewer 1 & $85.71(6 / 7)$ & $100(7 / 7)$ & $100(7 / 7)$ \\
Reviewer 2 & $85.71(6 / 7)$ & $42.86(4 / 7)$ & $85.71(6 / 7)$ \\
PPV (\%) & & & \\
Reviewer 1 & $94.74(18 / 19)$ & $100(29 / 29)$ & $100(29 / 29)$ \\
Reviewer 2 & $95.24(20 / 21)$ & $86.21(25 / 29)$ & $96.55(28 / 29)$ \\
NPV (\%) & & & \\
Reviewer 1 & $26.09(6 / 23)$ & $53.85(7 / 13)$ & $63.64(7 / 11)$ \\
Reviewer 2 & $28.57(6 / 21)$ & $23.08(3 / 13)$ & $46.15(6 / 13)$ \\
\hline
\end{tabular}

$N E T$, neuroendocrine tumors; $M R$, magnetic resonance; $D W I$, diffusion-weighted imaging; PPV, positive predictive value; NPV, negative predictive value.
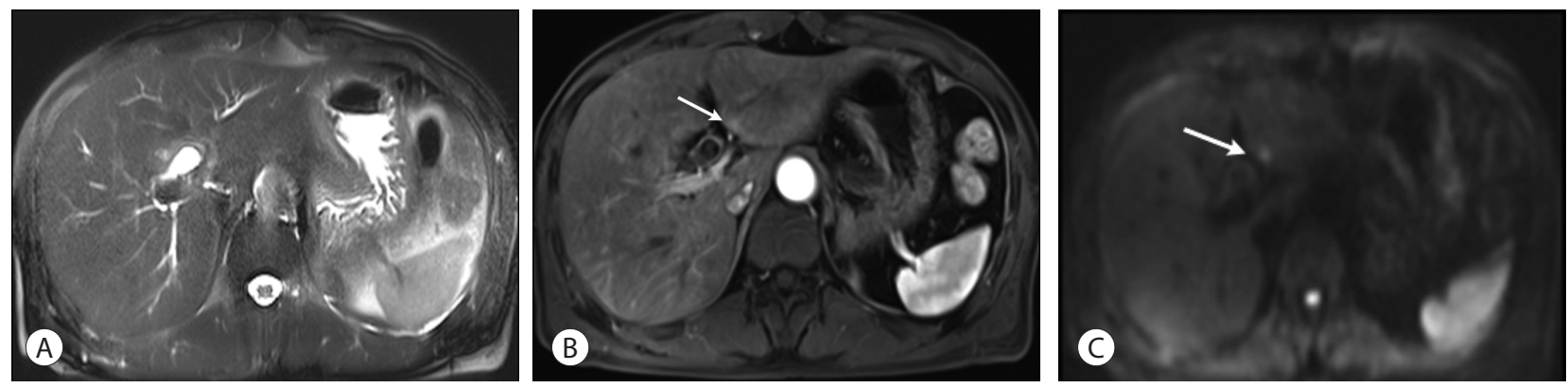

Figure 3. False negative on contrast-enhanced MRI. 62-year-old man with biopsy proven hepatic NET. (A) T2-weighted image shows no definite nodule in the liver. (B) Dynamic images using a fat-suppressed, 3D GRE sequence, on the arterial phase, demonstrates ill-defined small enhancing portion in the lateral segment of left lobe (arrow). (C) Diffusion weighted image using b values of $400 \mathrm{~s} / \mathrm{mm}^{2}$ clearly demonstrates small hyper signal intensity nodule in the lateral segment of left lobe (arrow). MRI, magnetic resonance imaging; NET, neuroendocrine tumors; GRE, gradient echo. 
Table 4. Sensitivity and specificity for detection of hepatic NET: lesion-by-lesion analysis for lesions smaller or equal to $1 \mathrm{~cm}$

\begin{tabular}{|c|c|c|c|}
\hline & \multicolumn{3}{|c|}{ Lesion-by-Lesion Analysis: $\leq 1 \mathrm{~cm}$ lesion } \\
\hline & $\begin{array}{l}\text { Precontrast } \\
\text { MR set }\end{array}$ & DWI set & $\begin{array}{c}\text { Combined } \\
\text { image set }\end{array}$ \\
\hline \multicolumn{4}{|l|}{ Sensitivity (\%) } \\
\hline Reviewer 1 & $10.75(10 / 93)$ & $50.54(47 / 93)$ & $65.59(61 / 93)$ \\
\hline Reviewer 2 & $15.05(14 / 93)$ & $39.78(37 / 93)$ & $61.29(57 / 93)$ \\
\hline \multicolumn{4}{|l|}{ Specificity (\%) } \\
\hline Reviewer 1 & $100(22 / 22)$ & $95.45(21 / 22)$ & $95.45(21 / 22)$ \\
\hline Reviewer 2 & $100(22 / 22)$ & $90.91(20 / 22)$ & $95.45(21 / 22)$ \\
\hline \multicolumn{4}{|l|}{ PPV (\%) } \\
\hline Reviewer 1 & $100(10 / 10)$ & $97.92(47 / 48)$ & $98.39(61 / 62)$ \\
\hline Reviewer 2 & $100(14 / 14)$ & $94.87(37 / 39)$ & $98.28(57 / 58)$ \\
\hline \multicolumn{4}{|l|}{ NPV (\%) } \\
\hline Reviewer 1 & $20.95(22 / 105)$ & $31.34(21 / 67)$ & $39.62(21 / 53)$ \\
\hline Reviewer 2 & $21.78(22 / 101)$ & $26.32(20 / 76)$ & $36.84(21 / 57)$ \\
\hline
\end{tabular}

NET, neuroendocrine tumors; MR, magnetic resonance; DWI, diffusion-weighted imaging; PPV, positive predictive value; NPV, negative predictive value.

the evaluation of hepatic NET and for most of the patients $(22 / 28)$ scanned on the $3 \mathrm{~T}$ system. Only six patients were scanned on a $1.5 \mathrm{~T}$ scanner. Third, as contrast-enhanced MRI was assessed in combination with DWI, the diagnostic effects of DWI and contrast-enhanced MRI when used independently could not be isolated. However, the image sets reflect clinical practice in which several imaging sequences are usually combined for the diagnostic evaluation.

In conclusion, combined DWI and contrast-enhanced MRI showed better performance for the detection of hepatic NET than the unenhanced MR protocol including DWI. However, DWI improved the diagnostic performance for detecting hepatic NET without statistical significance compared with the precontrast set. Therefore, DWI has the potential to be used as an alternative tool for the same purpose, and without using contrast material in patients with renal impairment.

\section{ACKNOWLEDGEMENT}

We would like to thank Bonnie Hami, MA (USA) for her editorial assistance in the preparation of this manuscript.

\section{Conflicts of Interest}

The authors have no conflicts to disclose.

\section{REFERENCES}

1. Berge T, Linell F. Carcinoid tumours. Frequency in a defined population during a 12-year period. Acta Pathol Microbiol Scand A 1976;84:322-330.

2. Frilling A, Sotiropoulos GC, Li J, Kornasiewicz O, Plöckinger U. Multimodal management of neuroendocrine liver metastases. HPB (Oxford) 2010;12:361-379.

3. Floriani I, Torri V, Rulli E, Garavaglia D, Compagnoni A, Salvolini $L$, et al. Performance of imaging modalities in diagnosis of liver metastases from colorectal cancer: a systematic review and metaanalysis. J Magn Reson Imaging 2010;31:19-31.

4. Kaewlai R, Abujudeh H. Nephrogenic systemic fibrosis. AJR Am J Roentgenol 2012;199:W17-W23.

5. Shimada K, Isoda H, Hirokawa Y, Arizono S, Shibata T, Togashi K. Comparison of gadolinium-EOB-DTPA-enhanced and diffusionweighted liver MRI for detection of small hepatic metastases. Eur Radiol 2010;20:2690-2698.

6. Ichikawa T, Haradome H, Hachiya J, Nitatori T, Araki T. Diffusionweighted MR imaging with a single-shot echoplanar sequence: detection and characterization of focal hepatic lesions. AJR Am J Roentgenol 1998;170:397-402.

7. Fukatsu H. ЗT MR for clinical use: update. Magn Reson Med Sci 2003:2:37-45.

8. Xu PJ, Yan FH, Wang JH, Lin J, Ji Y. Added value of breathhold diffusion-weighted MRI in detection of small hepatocellular carcinoma lesions compared with dynamic contrast-enhanced MRI alone using receiver operating characteristic curve analysis. J Magn Reson Imaging 2009;29:341-349.

9. Nasu K, Kuroki Y, Nawano S, Kuroki S, Tsukamoto T, Yamamoto S, et al. Hepatic metastases: diffusion-weighted sensitivity-encoding versus SPIO-enhanced MR imaging. Radiology 2006;239:122-130.

10. Takahara T, Kwee TC. Low b-value diffusion-weighted imaging: emerging applications in the body. J Magn Reson Imaging 2012;35:1266-1273.

11. Le Bihan D, Breton E, Lallemand D, Aubin ML, Vignaud J, LavalJeantet M. Separation of diffusion and perfusion in intravoxel incoherent motion MR imaging. Radiology 1988;168:497-505.

12. Chakraborty DP, Berbaum KS. Observer studies involving detection and localization: modeling, analysis, and validation. Med Phys 2004;31:2313-2330.

13. Zheng B, Chakraborty DP, Rockette HE, Maitz GS, Gur D. A comparison of two data analyses from two observer performance studies 
using Jackknife ROC and JAFROC. Med Phys 2005;32:1031-1034.

14. Proye C. Natural history of liver metastasis of gastroenteropancreatic neuroendocrine tumors: place for chemoembolization. World J Surg 2001;25:685-688.

15. Debray MP, Geoffroy O, Laissy JP, Lebtahi R, Silbermann-Hoffman 0 , Henry-Feugeas $M C$, et al. Imaging appearances of metastases from neuroendocrine tumours of the pancreas. Br J Radiol 2001;74:1065-1070.

16. van der Hoef M, Crook DW, Marincek B, Weishaupt D. Primary neuroendocrine tumors of the liver: MRI features in two cases. Abdom Imaging 2004;29:77-81.

17. Bader TR, Semelka RC, Chiu VC, Armao DM, Woosley JT. MRI of carcinoid tumors: spectrum of appearances in the gastrointestinal tract and liver. J Magn Reson Imaging 2001;14:261-269.

18. Dromain C, de Baere T, Baudin E, Galline J, Ducreux M, Boige V, et al. MR imaging of hepatic metastases caused by neuroendocrine tumors: comparing four techniques. AJR Am J Roentgenol 2003;180:121-128.

19. Soyer $P$, Gueye $C$, Somveille E, Laissy JP, Scherrer A. MR diagnosis of hepatic metastases from neuroendocrine tumors versus heman- giomas: relative merits of dynamic gadolinium chelate-enhanced gradient-recalled echo and unenhanced spin-echo images. AJR Am J Roentgenol 1995;165:1407-1413.

20. Chung WS, Kim MJ, Chung YE, Kim YE, Park MS, Choi JY, et al. Comparison of gadoxetic acid-enhanced dynamic imaging and diffusion-weighted imaging for the preoperative evaluation of colorectal liver metastases. J Magn Reson Imaging 2011;34:345-353.

21. Kim YK, Kim CS, Han YM, Lee YH. Detection of liver malignancy with gadoxetic acid-enhanced MRI: is addition of diffusionweighted MRI beneficial? Clin Radiol 2011;66:489-496.

22. Holzapfel K, Eiber MJ, Fingerle AA, Bruegel M, Rummeny EJ, Gaa J. Detection, classification, and characterization of focal liver lesions: value of diffusion-weighted MR imaging, gadoxetic acid-enhanced MR imaging and the combination of both methods. Abdom Imaging 2012;37:74-82.

23. Kenis C, Deckers F, De Foer B, Van Mieghem F, Van Laere S, PouilIon M. Diagnosis of liver metastases: can diffusion-weighted imaging (DWI) be used as a stand alone sequence? Eur J Radiol 2012;81:1016-1023.

Appendix 1. MR parameters

\begin{tabular}{|c|c|c|c|c|}
\hline MR scanner & FS T2WI & T1W GRE & T2 HASTE & FS-3D T1W GRE \\
\hline \multicolumn{5}{|c|}{ Signa Excite HDX $(n=6)$} \\
\hline TR (msec) & 2,000 & 170 & 730 & 5.3 \\
\hline TE (msec) & 85 & 2.0/4.2 (OP/IP) & 160 & 2.6 \\
\hline ETL & 30 & 1 & 1 & 1 \\
\hline FA & $90^{\circ}$ & $60^{\circ}$ & $90^{\circ}$ & $12^{\circ}$ \\
\hline Slab thickness & 7 & 7 & 7 & $4.8-6.0$ \\
\hline FOV (mm) & $370-370$ & $350-350$ & $350-350$ & $350-350$ \\
\hline Matrix & $256 \times 256$ & $320 \times 224$ & $320 \times 256$ & $384 \times 192$ \\
\hline \multicolumn{5}{|l|}{ Verio $(n=20)$} \\
\hline TR (msec) & 6,550 & 4.0 & 980 & 3.4 \\
\hline TE (msec) & 95 & 1.3/2.2 (OP/IP) & 145 & 1.2 \\
\hline ETL & 256 & 1 & 256 & 1 \\
\hline FA & $130^{\circ}$ & $9^{\circ}$ & $130^{\circ}$ & $11^{\circ}$ \\
\hline Slab thickness & 7 & 3 & 8 & $3-3.6$ \\
\hline $\mathrm{FOV}(\mathrm{mm})$ & $380-380$ & $380-380$ & $380-380$ & $380-380$ \\
\hline Matrix & $448 \times 269$ & $320 \times 285$ & $384 \times 307$ & $384 \times 307$ \\
\hline \multicolumn{5}{|l|}{ Achieva $(n=2)$} \\
\hline $\mathrm{TR}$ (msec) & 2,087 & 3.5 & 1,992 & 3.1 \\
\hline TE (msec) & 160 & 1.2/2.3 (OP/IP) & 80 & 1.5 \\
\hline ETL & 92 & 162 & 95 & 46 \\
\hline FA & $90^{\circ}$ & $12^{\circ}$ & $90^{\circ}$ & $10^{\circ}$ \\
\hline Slab thickness & 5 & 5 & 5 & 4 \\
\hline FOV (mm) & $340-340$ & $340-340$ & $340-340$ & $340-340$ \\
\hline Matrix & $252 \times 270$ & $144 \times 174$ & $252 \times 270$ & $228 \times 230$ \\
\hline
\end{tabular}

MR, magnetic resonance; FS, fat suppressed; T2W1, T2-weighted imaging; T1W GRE, T1-weighted gradient echo; T2 HASTE, T2 half-Fourier acquisition singleshot turbo spin-echo; TR, repetition time; TE, echo time; OP, opposed-phase; IP, in-phase; ETL, echo train length; FA, flip angle; FOV, field of view. 